\title{
LA FALACIA DE LA RESISTENCIA FRANCESA Y NUESTRA COTIDIANA ILUSIÓN DE MORAL
}

\section{THE FALLACY OF FRENCH RESISTANCE AND OUR EVERYDAY ILLUSION OF MORAL}

\section{Jorge VILLALBA-ARIAS ${ }^{1}$, Julio TORALES ${ }^{1}$.}

${ }^{1}$ Universidad Nacional de Asunción, Facultad de Ciencias Médicas, Cátedra de Psiquiatría, San Lorenzo, Paraguay.

Cómo citar este artículo: Villalba-Arias J, Torales J. La falacia de la resistencia francesa y nuestra cotidiana ilusión de moral. Med. clín. soc. 2019;3(1):19-26.

\section{RESUMEN}

En cada instante de nuestra existencia consciente, nuestra mente es bombardeada por más de 11 millones de inputs de información. Debido a que nuestro cerebro dispone de apenas 10 Watts de energía, es comprensible que el mismo deba integrar estrategias que lo lleven a economizar recursos, no solo con el objetivo de optimizar recursos energéticos, sino también de asegurar respuestas que sean lo suficientemente rápidas como útiles e incluso vitales para la subsistencia. Tal situación es posible gracias a los heurísticos. En este artículo damos un recorrido por los principales heurísticos para luego explicar los sesgos que se generan a través de ellos. Dichos sesgos comprometen severamente nuestra objetividad en la toma de decisiones por lo cual incrementan la subjetividad de la moral y aleja a esta última de lo ético.

Palabras clave: Heurístico; Sesgo; Falacia; Moral; Ética.

\section{ABSTRACT}

At every moment of our conscious existence, our mind is bombarded by more than 11 million information inputs. Because our brain has only 10 Watts of energy, it is understandable that it should integrate strategies that lead to economize resources, not only with the aim of optimizing energy resources, but also to ensure responses that are fast enough and useful. and even vital for subsistence. Such a situation is possible thanks to the heuristics. In this article we give a tour of the main heuristics to then explain the biases that are generated through them. These biases severely compromise our objectivity in decision-making, increase the subjectivity of morality and move morality away from ethics.

Keywords: Heuristic; Bias; Fallacy; Moral; Ethics.

\section{INTRODUCCIÓN}

Ya sea por la limitada disponibilidad energética para nuestro cerebro, o porque no pocas veces disponemos de toda la información que desearíamos o porque nos embarga la incertidumbre de las consecuencias de tomar una u otra decisión, en muchas ocasiones tomamos "atajos" mentales para obtener respuestas a los problemas. Estos atajos mentales que tomamos de forma inconsciente, en psicología se llaman "Heurísticos", y nos ayudan a simplificar la gran cantidad de procesos mentales que llevamos a cabo constantemente y a hacer más llevadera nuestra vida diaria (1-3). 
Y es que nuestro cerebro no es capaz de procesar toda la información que recibe a través de los sentidos, por lo que necesita discriminar a cuáles datos dar mayor prioridad sobre otros. Cuando nuestros atajos mentales o heurísticos nos conducen a errores de conclusión, les llamamos sesgos cognitivos (1).

Es nuestra esperanza que las autoridades de las escuelas de Medicina decidan tomar las medidas necesarias para transformar esas instituciones en entornos seguros donde los futuros médicos, fisioterapeutas, enfermeros y otros, puedan prosperar y tener éxito no solo en sus carreras profesionales, sino también en sus vidas personales.

\section{UN ÓRGANO CON DECISIONES SUMAMENTE COMPLEJAS Y RECURSOS MUY LIMITADOS}

De los 11 millones de inputs de información que llegan a nuestro cerebro en cada instante de consciencia, solo 40 de ellos son abordables cabalmente y analizables para una respuesta calculada y, de alguna manera considerable como propia; los más de 10 millones de inputs restantes son evaluados de manera automática y, las respuestas a ellas son decididas por complejos mecanismos heurísticos de los que apenas tomamos consciencia (1).

A partir de esta premisa surge necesariamente la duda de quiénes somos realmente y, qué tan nuestras son las decisiones que tomamos a diario; o, en todo caso, qué parte de nosotros es real y auténticamente nuestro y, qué parte de nosotros es el producto de lo asimilado del ambiente (2).

Para tratar de comprender la manera en la que un aparato de 10 Watts de disponibilidad energética (el cerebro), se hace cargo de la vasta cantidad de información que a él llega de manera constante, es necesario tomar comprender los heurísticos mentales y cómo estos generan los sesgos cognitivos (3).

En sus orígenes, la heurística comparte etimología con eureka; provienen del griego y significan hallar, inventar (4). Con el paso del tiempo fue adoptando múltiples acepciones, a tal punto que su significado varía según se lo emplee como sustantivo o adjetivo. La acepción que nos interesa fue introducida por Albert Einstein en su publicación de 1921, la misma que le daría el premio Nobel en Física por sus postulados sobre el efecto fotoeléctrico. Aun así, quien lo haría conocido sería el matemático húngaro George Pólya a través de su libro Cómo Resolverlo (How to solve it), en el cual se aboca a tratar de descubrir cómo hacen los experimentados matemáticos para encontrar soluciones a problemas (5).

Pero, ¿qué son los heurísticos? Los heurísticos son un conjunto de reglas metodológicas no necesariamente forzosas, positivas y negativas, que sugieren o establecen cómo proceder y qué problemas evitar a la hora de generar soluciones y elaborar hipótesis (6). Si bien, dicho concepto puede parecer complejo, los heurísticos tienen como fin exactamente lo opuesto. $Y$ eso es porque los heurísticos son esquemas mentales que se utilizan a modo de atajo con el objeto de dar soluciones con el menor consumo de tiempo y de recursos mentales. Son esquemas mentales fundamentales para nuestro funcionamiento diario, pero al ser automáticos son susceptibles al ambiente y a la intervención de terceros (7). Cuando al inicio hablábamos de que solo 40 de los datos externos recibidos por nuestros sentidos son analizados antes de 
dar una respuesta a ellos, ahora vemos que el resto de los datos recibidos que requieran respuesta serán evaluados y solucionados de manera "automática" por nuestro cerebro a través de los heurísticos $(1,6,7)$.

Entre estos heurísticos se encuentra el Heurístico de representatividad. El mismo establece que cuanto más típico es un caso concreto respecto a un modelo, mayor es la probabilidad subjetiva de que el caso pertenezca al modelo y, por tanto, más probable que asignemos el caso a esa categoría (8). El problema con este heurístico es que basa su respuesta en características observables y deja de lado cuestiones como la probabilidad estadística (que es mucho más fiable a la hora de determinar un resultado), a la hora de realizar el análisis (9). Por ejemplo: es usual que un docente se sorprenda cuando un alumno que tiene buen rendimiento en las prácticas tiene un pobre rendimiento en los exámenes (si uno realizase un análisis más profundo de este ejemplo, uno comprendería que los criterios evaluativos de una práctica no siempre se condicen con los criterios evaluativos de una prueba parcial por lo que un buen rendimiento en uno no necesariamente implica un buen rendimiento en lo otro).

Otro ejemplo es el que se da cuando visitamos un hospital: si vemos a un hombre en dicho contexto, y observamos que porta unos tubos para recolección de muestras biológicas en la mano y unos bolígrafos en el bolsillo de la chaqueta blanca, lo más probable es que asumamos que se trata de un médico cuando, en realidad, hay más probabilidades de que sea un enfermero (porque los hay en mayor cantidad en un hospital cuando comparados con los médicos) o incluso, podría tratarse de un técnico de laboratorio, o un doctor en bioquímica. Una vez más el cerebro prioriza en su análisis las características de similitud entre lo que observa y lo que tiene computado en la memoria como modelo de una profesión (en este caso un médico), para determinar su respuesta; $y$, debido al poco tiempo para análisis desestima la probabilidad y posibilidad de que en realidad sea un enfermero o un personal de laboratorio. Las consecuencias de este tipo de análisis son relativas a las funciones que uno desempeña; no es lo mismo que una persona en calidad de familiar de paciente confunda a un enfermero con un médico al momento de solicitar indicaciones para llegar a la habitación de su familiar paciente, que el que un guardia de seguridad asuma que una persona es un médico solo porque lleva puesto una chaqueta blanca y por ello le permita acceder a una zona cuyo acceso es exclusivo para médicos (una persona extraña a la institución podría adquirir una chaqueta blanca en una tienda y hacerse pasar por médico y con ello comprometer la seguridad del hospital, por ejemplo).

El heurístico de disponibilidad establece que nuestras estimaciones respecto a eventos se basan en el conocimiento obtenido a través de nuestras vivencias de ese evento (10). Por ejemplo, personas que viven en una zona de extrema pobreza calcularían que la esperanza de vida se sitúa cerca de los 55 años, cuando en realidad, la esperanza de vida global actualmente supera los 75 años (11). Otra manera de ver al Heurístico de disponibilidad es que, cuanto más fácil nos resulta recordar un evento, más probable nos parece su ocurrencia. Por ejemplo, si se nos pregunta si los cocos o los tiburones causan más lesiones anualmente, lo más probable es que respondamos que son los tiburones cuando se ha visto que los ataques de tiburón rara vez alcanzaron una incidencia de 10 personas al año mientras que llegaron a registrarse 150 personas lesionadas por caídas de coco (12). Este error en particular se da debido a que la prensa da mayor relevancia a ataques de tiburón que a lesiones causadas por caída de cocos. 
El heurístico de ajuste y anclaje ayuda a las personas a realizar estimaciones numéricas y juicios cuantitativos en contextos de incertidumbre (13). En otras palabras, cuando nos encontramos ante un problema sobre el cual no tenemos información previa, utilizaremos las primeras informaciones que nos vayan proveyendo al respecto para usarlos como lineamientos en el tema (independientemente de que tales datos representen o no una tendencia en dicho asunto). Como ejemplo, utilizaremos un experimento liderado por los psicólogos Daniel Kahneman (Premio Nobel de Economía en el año 2002) y Amos Tversky y que fuera realizado por primera vez en 1973. En dicho experimento, llevado a cabo en Estados Unidos de América (14), se pidió a un grupo de personas que escribieran los cuatro últimos dígitos de su número de la seguridad social en un trozo de papel antes de estimar la edad que tenía George Washington cuando murió. Los investigadores pensaron que este número aleatorio podría servir como un número de anclaje. En línea con esa predicción, lo que encontraron fue que aquellos participantes con números de la seguridad social altos estimaban que había muerto con mayor edad que aquellos con números bajos.

Estos heurísticos citados más arriba son los responsables de los sesgos cognitivos $(15,16)$. Los sesgos cognitivos son fenómenos que causan una alteración en el procesamiento de la información captada por nuestros sentidos, lo cual genera una distorsión, juicio errado, interpretación incoherente o ilógica sobre el fundamento de la información de que disponemos (17, 18).

Son varios los sesgos cognitivos que pueden generarse, pero el objeto del presente artículo es el de ilustrar los mecanismos a través de los cuales una postura moral puede estar basada en prejuicios más que en datos objetivos (estén estos datos disponibles o no); por ello nos abocaremos a los siguientes:

- El sesgo de correspondencia: también conocido como error fundamental de atribución, se refiere a la disposición que tiene uno de atribuir los logros a factores internos (inteligencia, preparación, dedicación, etcétera), mientras que atribuimos las "desgracias" a factores externos (mala suerte, desventajas sociales, económicas, etcétera). Cabe destacar que el error fundamental de atribución adquiere características opuestas cuando aplicada a un tercero; así, cuando se evalúa a una persona que no seamos nosotros, tenderemos a atribuir sus logros a factores externos mientras que sus fracasos a factores internos (19).

- El efecto Forer: es la condición en la que consideramos que descripciones por demás vagas y generales son percibidas como específicas y exclusivas cuando dirigidas hacia uno mismo. Por ejemplo: los horóscopos y cartas astrales (20).

- El sesgo egocéntrico determina que nos atribuimos mayor protagonismo al resultado de una acción conjunta del que nos daría un observador externo. A diferencia del sesgo de correspondencia, en el sesgo egocéntrico la auto-atribución es indistinta al matiz positivo o negativo de la situación (21).

- El efecto halo predispone a que se tome una característica dada de un individuo para extrapolar sus cualidades en otras características que no guardan relación con la primera. Por ende, si nos sentimos atraídos por alguien, sería natural pensar que, por ser una persona bella también 
sea una hermosa persona. Otro ejemplo es la aplicación de este sesgo por parte de marcas reconocidas; Mercedes - Benz es mundialmente reconocido por la calidad de sus automóviles, por lo cual se asume que sus llaveros o lentes de sol deben gozar de las mismas cualidades (1, 22).

- La ilusión de entendimiento asimétrico nos da la falsa impresión de que tenemos mayor insight del que consideramos tienen los demás de sí mismos. Se parece en algo a la ilusión de transparencia, aunque esta última se refiere al hecho de considerar que los demás conocen mucho más nuestra personalidad de lo que en realidad lo hacen (23).

- El fenómeno del mundo justo conlleva la nefasta presunción de que las tragedias ocurren en respuesta a actos ruines o solo a personas malas. Existen estudios que demuestran que personas con una visión de mundo justo asumen que si una mujer fue violada es porque la misma probablemente lo buscó al vestirse de forma provocativa. Igualmente es debido a esta visión sesgada que muchos consideran que a todo acto vil le sobreviene eventualmente un castigo justo (24).

- El sesgo de confirmación lleva a la selección inconsciente de datos que favorezcan nuestras teorías y a la desestimación de aquellas que se opongan a las mismas; cuando se encuentran datos ambiguos, se los toma como favorables a nuestras teorías (25-27).

- El sesgo retrospectivo se torna evidente en aquellas ocasiones en las que vemos un filme de misterio por segunda vez; una vez resuelto el misterio una primera vez, la segunda vez que se evalúan las pistas se torna mucho más fácil predecir con justificación deductiva quién sería el personaje detrás del misterio $(28,29)$.

Si analizamos con el suficiente detenimiento cada uno de estos sesgos, nos resulta imposible negar lo lábil de nuestras supuestas posturas objetivas, entre ellas, las relativas a la moral. Más aún cuando sabemos que la moral tiene como base fundamental las costumbres del grupo al que uno pertenece (30-32).

Pero existe la ética, la cual es considerada como la moral transcultural, ya que se espera que la misma analice los hechos desarraigándose de factores socioculturales, buscando entender las bondades o maldades de los actos (33-35).

La comprensión de lo referente a los sesgos cognitivos nos ubica en la incómoda posición de aceptar que es inherente a nuestro sistema de análisis de la realidad el "Ilevar agua a nuestro molino". Pero tal realidad no debería exentarnos del esfuerzo por buscar objetividad en cada acto. $Y$, si bien es cierto que la inmensa mayoría de los inputs de datos que reciba nuestra mente será evaluada por los heurísticos ( $y$, por ende, tendrán respuestas que, en gran medida, podríamos considerar más propias de nuestra realidad sociocultural más que individual), debemos poner empeño en identificar aquellos inputs cuyas decisiones generen repercusiones trascendentales en nosotros, los nuestros o la sociedad. Esto último, debido a que, como ya se mencionó con anterioridad, nuestras decisiones automáticas están supeditadas a lo establecido por la cultura y/o por lo inducido por terceras personas. Es así como políticos y líderes 
influencian nuestras decisiones e incluso la imagen que tenemos de ellos a través de los medios de comunicación masiva. Tales influencias sobre nuestros heurísticos incluso determinan nuestros gustos e intereses; de ahí la importancia que se ha dado al marketing en los últimos tiempos $(1-3,36,37)$.

No es fortuito el que se haya dejado en el tintero en este artículo a la fantasía de la resistencia francesa. $Y$ eso es porque se ha visto que es inherente a nuestra estructura cognitiva el creernos exentos de la influencia de los heurísticos y los sesgos que estos producen. Vale aclarar que esta falacia (de la fantasía de la resistencia francesa) expresa que: cuando evaluamos si hubiéramos vivido la invasión de Europa por parte de la Alemania Nazi, tendemos a considerar que nosotros habríamos formado parte de la Resistencia Francesa, cuando, en realidad, desde la perspectiva de la probabilidad estadística y los rasgos de personalidad, es más probable que no hubiéramos formado parte de dicho grupo (38).

Es por ello que la fantasía de la resistencia francesa es uno de los sesgos más peligrosos con los que lidiamos a diario. Y eso debido a que, cuando pensamos que las decisiones que estamos tomando son nuestras más que un producto de las influencias de la sociedad o los líderes, lo más probable es que estemos siendo víctimas de esta falacia antes que verdaderos evaluadores de los motivos y justificaciones que soportan nuestras decisiones $(1,9,14,38)$.

\section{CONCLUSIÓN}

En definitiva, nuestro cerebro hace uso de atajos mentales para tomar decisiones de forma más rápida y ser así, más ágil a la hora de reaccionar, desenvolverse y adaptarse al entorno. De esta forma, se generan una serie de estrategias mentales que nos permiten aumentar nuestras probabilidades de supervivencia (1).

Hemos nombrado algunos de los sesgos cognitivos más comunes y los hemos explicado, pero, ¿ser conocedores de los mismos es suficiente para evitar caer en ellos? Lamentablemente no. Nuestro cerebro seguirá haciendo uso (afortunadamente), de heurísticos o atajos mentales e, inevitablemente, algunas veces derivarán en errores que, de forma no consciente, también afectarán a nuestra toma de decisiones. A veces podremos reconocerlos, pero la mayoría de las veces, no seremos capaces $(1,10,38)$.

\section{CONFLICTOS DE INTERÉS Y FUENTE DE FINANCIACIÓN}

Los autores declaran no poseer conflictos de interés. Fuente de financiación: ninguna.

\section{REFERENCIAS BIBLIOGRÁFICAS}

1. Cortada de Kohan N. Los Sesgos Cognitivos en la Toma de Decisiones. International Journal of Psychological Research 2008;1(1):68-73. URL.

2. Pfister HR, Böhm, G. The multiplicity of emotions: A framework of emotional functions in decision making. Judgment and Decision Making 2008;3(1):5-17. URL.

3. Crotty P, Sangrey T, Levy WB. 2006. 'Metabolic Energy Cost of Action Potential Velocity'. J Neurophysiol. 2006;96(3):1237-46. https://doi.org/10.1152/in.01204.2005

4. Beuchot M. Heurística y hermenéutica. 1a ed. Ciudad de México: Editorial de la UNAM; 1999. 
5. Jeng M. A selected history of expectation bias in physics. Am. J. Phys. 2006;74:578. https://doi.org/10.1119/1.2186333

6. Myers DG. Social psychology. $10^{\text {th }}$ ed. New York: McGraw-Hill; 2009.

7. Hansen HV. The Straw Thing of Fallacy Theory: The Standard Definition of 'Fallacy'. Argumentation 2002;16(2):133-155. https://doi.org/10.1023/A:1015509401631

8. Kahneman D, Frederick S. Representativeness revisited: Attribute substitution in intuitive judgment. In: Gilovich T, Griffin D, Kahneman D, editors. Heuristics and Biases. The Psychology of Intuitive Judgment. New York: Cambridge University Press; 2012. p. 49-81.

9. Kahneman D, Slovic P, Tversky A. Judgment under uncertainty: Heuristics and biases. New York: Cambridge University Press; 1982.

10. Slovic $P$, Finucane $M$, Peters $E$, MacGregor DG. The affect heuristic. In: Gilovich T, Griffin D, Kahneman D, editors. Heuristics and Biases. The Psychology of Intuitive Judgment. New York: Cambridge University Press; 2012. p. 397-420.

11. Restrepo LF, Rodríguez H. Análisis comparativo de la esperanza de vida en Sudamérica, 1980 - 2010. Rev Univ. salud. 2014;16(2): 177-187. URL.

12. Pinker S. The truth about falling coconuts. CMAJ. 2002;166(6):801. URL.

13. Chapman GB, Johson EJ. The Limits of Anchoring. Journal of Behavioral Decision Making. 1994;7(4):223-242. https://doi.org/10.1002/bdm.3960070402

14. Tversky A, Kahneman D. Judgment under uncertainty: Heuristics and biases. Science 1974;1185(4157):1124-1131. URL.

15. Worchel S, Cooper J, Geothals GR, Olson JM. Psicología Social. México: International Thomson Ediciones; 2002.

16. Haselton MG, Nettle D, Andrews PW. The evolution of cognitive bias. In: Buss DM, editor. The Handbook of Evolutionary Psychology. Hoboken: John Wiley \& Sons; 2005. p. 724-746.

17. Busenitz LW, Lau CM. A Cross-Cultural Cognitive Model of New Venture Creation. Entrepreneurship Theory and Practice 1996;20(4):25-40. https://doi.org/10.1177/104225879602000403

18. Baron RA. Cognitive mechanisms in entrepreneurship: Why and when entrepreneurs think differently than other people. Journal of Business Venturing 1998;13:275-294.

19. Gilbert DT. Speeding with Ned: A personal view of the correspondence bias. In: Darley JM, Cooper J, editors. Attribution and social interaction: The legacy of E. E. Jones. Washington: APA Press; 1998.

20. Forer BR. The fallacy of personal validation: A classroom demonstration of gullibility. Journal of Abnormal and Social Psychology 1949;44(1):118-123. https://doi.org/10.1037/h0059240

21. Baron J. Thinking and deciding. $3^{\text {rd }}$ ed. New York: Cambridge University Press; 2000.

22. Nisbett R, Wilson TD. The halo effect: Evidence for unconscious alteration of judgments. Journal of Personality and Social Psychology 1977;35(4):250-256. https://doi.org/10.1037/0022-3514.35.4.250

23. Pronin E, Kruger J, Savitsky K, Ross. You don't know me, but I know you: the illusion of asymmetric insight. Journal of Personality and Social Psychology. 2001;81(4):639-656. https://doi.org/10.1037/0022-3514.81.4.639

24. Zubieta E, Barreiro A. Percepción social y creencia en el mundo justo. Un estudio con estudiantes argentinos. Revista de Psicología . 2006;XXIV(2):175-196. URL. 
25. Sternberg RJ, Roediger III HL, Halpern DF, editors. Critical thinking in psychology. New York: Cambridge University Press; 2007.

26. Shermer M. The Political Brain [Internet]. Armonk, New York: Scientific American; 1 July 2006 [cited 28 December 2018]. Available from: https://www.scientificamerican.com/article/the-political-brain/

27. Emerson GB, Warme WJ, Wolf FM, Heckman JD, Brand RA, Leopold SS. Testing for the Presence of Positive-Outcome Bias in Peer Review: A Randomized Controlled Trial. Arch Intern Med. 2010;170(21):1934-1939. https://doi.org/10.1001/archinternmed.2010.406

28. Fischhoff $B$. Hindsight $\neq$ foresight: The effect of outcome knowledge on judgment under uncertainty. Qual Saf Health Care. 2003;12(4):304-312. https://doi.org/10.1136/qhc.12.4.304

29. Arkes H, Faust D, Guilmette TJ, Hart K. Eliminating Hindsight Bias. Journal of Applied Psychology 1988;73(2):305-307. URL.

30. Korstanje M. El miedo político bajo el prisma de Hannah Arendt. Revista SAAP 2014;8(1):99-126. URL.

31. Haidt J, Graham J. When Morality Opposes Justice: Conservatives Have Moral Intuitions that Liberals may not Recognize. Social Justice Research. 2007;20(1):98-116. https://doi.org/10.1007/s11211-007-0034-z

32. Chapouthier G. To what extent is moral judgment natural? European Review (GB) 2004;12(2):179-183.

33. Kidder R. How Good People Make Tough Choices: Resolving the Dilemmas of Ethical Living. New York: Harper Collins; 2003.

34. Richard, P, Linda E. The Miniature Guide to Understanding the Foundations of Ethical Reasoning. United States: Foundation for Critical Thinking Free Press; 2006.

35. Olson RG. Deontological Ethics. In: Edwards P, editor. The Encyclopedia of Philosophy. London: Collier Macmillan; 1967. p. 343.

36. Scholl JA, Mederer HJ, Scholl RW. Leadership, Ethics, and Decision-Making. In: Farazmand A, editor. Global Encyclopedia of Public Administration, Public Policy, and Governance. New York: Springer: 2016. p. 1-11. https://doi.org/10.1007/978-3-31931816-5 2407-1

37. Selart M, Johansen ST. Ethical Decision Making in Organizations: The Role of Leadership Stress. Journal of Business Ethics. 2011:99(2); 129-143. https://doi.org/10.1007/s10551-010-0649-0

38. Schulz K. En defensa del error. Madrid: Ed. Siruela; 2015 